\title{
Subcompartmented oxphosomic model of the mitochondrial oxidative phosphorylation system
}

\author{
Ukolova I.V. \\ Siberian Institute of Plant Physiology and Biochemistry SB RAS, Irkutsk, Russia \\ *email: irina@sifibr.irk.ru
}

Mitochondria are vital organelles that provide the cells of aerobic eukaryotic organisms with energy in the form of ATP via oxidative phosphorylation system (OXPHOS). OXPHOS enzymes (complexes I-V) are located in the inner mitochondrial membrane, mainly in cristae domain. At present, there is a large amount of experimental data indicating that the respiratory complexes $\mathrm{I}_{1} \mathrm{III}_{2}$ and IV in vivo can physically interact in different stoichiometry, thereby forming supercomplexes (SCs). The current models of supramolecular organisation of OXPHOS are contradictory and suggest either highly ordered respiratory strings or, vice versa, a set of randomly dispersed respiratory complexes and SCs. As for ATP synthase (also called as complex V), it is assumed that it does not form associations with respiratory complexes and SCs and works autonomously. Recently, while studying the OXPHOS arrangement in pea shoot mitochondria, we found for the first time, that complex IV and ATP synthase can interact and form the $\mathrm{IV}_{1} \mathrm{Va}_{2}$ supercomplex (Ukolova et al., 2020). In addition to this SC, other new structures, that had not been detected earlier, were also revealed. These findings suggested that all enzymes of the OXPHOS system in vivo could form a single superstructure called as 'oxphosome'. Taking into account phylogenetic conservation of supercomplex organisation as well as our findings and currently available highresolution structures of respiratory complexes and SCs, a subcompartmented oxphosomic model of the mitochondrial energy transduction system has been developed. According to this model, a significant part of the OXPHOS complexes form oxphosomes, which are located mainly in the cristal subcompartment of mitochondria as highly organised 'strings' or 'patches', which are in fact 'mini factories' for ATP production. Such organisation significantly increases the efficiency of the OXPHOS system, since it enables the use of not only protons of the bulk phase of the intermembrane space, but also protons associated with the membrane and produced in high local concentrations by respiratory enzymes of oxphosomes. 